\title{
Investigating the 1-year decline in midbrain-to-pons ratio in the differential diagnosis of PSP and IPD
}

\author{
Silja Kannenberg ${ }^{1} \cdot$ Julian Caspers $^{2} \cdot$ Lars Dinkelbach $^{1} \cdot$ Alexia-S. Moldovan $^{1,3} \cdot$ Stefano Ferrea $^{1} \cdot$ \\ Martin Südmeyer $^{1,4} \cdot$ Markus Butz $^{1}$. Alfons Schnitzler ${ }^{1}$. Christian J. Hartmann ${ }^{1,3}$
}

Received: 18 June 2020 / Revised: 10 November 2020 / Accepted: 19 November 2020 / Published online: 4 December 2020

(C) The Author(s) 2020

\begin{abstract}
Background A reliable measure of PSP-specific midbrain atrophy, the midbrain-to-pons ratio (MTPR) has been reported to support the differential diagnosis of progressive supranuclear palsy (PSP) from idiopathic Parkinson's disease (IPD). Since longitudinal analyses are lacking so far, the present study aimed to evaluate the diagnostic value of the relative change of MTPR (rel $\Delta$ t_MTPR) over a 1-year period in patients with PSP, IPD, and healthy controls (HC).

Methods Midsagittal individual MRIs of patients with PSP $(n=15)$, IPD $(n=15)$, and healthy controls (HC; $n=15)$ were assessed and the MTPR at baseline and after 1 year were defined. The diagnostic accuracy of the MTPR and its relative change were evaluated using ROC curve analyses.

Results PSP-patients had a significantly lower MTPR at baseline $(M=0.45 \pm 0.06)$, compared to both non-PSP groups (F $(2,41)=62.82, p<0.001)$, with an overall predictive accuracy of $95.6 \%$ for an MTPR $\leq 0.54$. PSP-patients also presented a significantly stronger 1-year decline in MTPR compared to IPD $(p<0.001)$. Though predictive accuracy of rel $\Delta_{\mathrm{t}-}$ MTPR for PSP $(M=-4.74 \% \pm 4.48)$ from IPD $(M=+1.29 \pm 3.77)$ was good $(76.6 \%)$, ROC analysis did not reveal a significant improvement of diagnostic accuracy by combining the MTPR and rel $\Delta_{\mathrm{t}-}$ MTPR $(p=0.670)$. Still, specificity for PSP increased, though not significantly $(p=0.500)$.

Conclusion The present results indicate that the rel $\Delta_{\mathrm{t}-}$ MTPR is a potentially useful tool to support the differential diagnosis of PSP from IPD. For its relative 1-year change, still, more evaluation is needed.
\end{abstract}

Keywords Progressive supranuclear palsy $\cdot$ Idiopathic Parkinson's disease $\cdot$ Midbrain-to-pons ratio $\cdot$ Atypical parkinsonism · MRI

Julian Caspers

julian.caspers@med.uni-duesseldorf.de

1 Institute of Clinical Neuroscience and Medical Psychology, Medical Faculty, Heinrich Heine University Düsseldorf, Universitätsstraße 1, 40225 Düsseldorf, Germany

2 Department of Diagnostic and Interventional Radiology, Medical Faculty, University Hospital Düsseldorf, Moorenstraße 5, 40225 Düsseldorf, Germany

3 Department of Neurology, Medical Faculty, University Hospital Düsseldorf, Moorenstraße 5, 40225 Düsseldorf, Germany

4 Department of Neurology, Ernst Von Bergmann Hospital, Charlottenstraße 72, 14467 Potsdam, Germany

\section{Introduction}

The differential diagnosis of progressive supranuclear palsy (PSP) from Parkinson's disease (IPD) is not trivial [1, 2]. At present, the clinical diagnosis of PSP is primarily based on identifying disease-specific symptoms, which may not have fully developed in early stages of the disease [3, 4]. Accordingly, misdiagnoses occur frequently due to a substantial overlap of symptoms [5]. Still, a more rapid progression and an overall poor prognosis in PSP underline the clinical need for objective biomarkers to facilitate early and precise diagnosis $[6,7]$.

As specific brain structures are known to be atrophic to different extents in different Parkinsonian diseases, diseasespecific alterations detectable by structural magnetic resonance imaging (MRI) were suggested to support the diagnosis of PSP [8-13]. 
A hallmark, known to be highly specific of PSP, is midbrain atrophy $[14,15]$. Hence, the so-called midbrain-topons ratio (MTPR) was introduced as a potential biomarker to distinguish between PSP and IPD and constitutes a reliable method to quantify PSP-specific midbrain atrophy [16-18]. Since PSP progresses considerably faster than IPD, PSP-specific rates of atrophy can potentially serve as biomarkers of the disease and support differential diagnosis $[19,20]$.

To substantiate this notion, we aimed to evaluate the diagnostic value of the MTPR and its relative change (rel $\Delta_{\mathrm{t}-}$ MTPR) over time. We analyzed structural MRI scans at a 1-year interval and defined the MTPR in patients with PSP and IPD as well as healthy controls (HC).

\section{Methods}

\section{Participants}

The study included 15 patients with probable or possible PSP, 15 patients with IPD as well as 15 HC. Trained movement disorders specialists (CJH; MS) confirmed clinical diagnoses of PSP and IPD, based on the NINDS diagnostic criteria [21]. Additionally, the MDS diagnostic criteria were applied, retrospectively to every patient [22]. Clinical records were reviewed, and groups were matched for age and disease duration (DD) at baseline (BL). The study was approved by the local ethics committee (study no. 2849). All participants gave prior written informed consent and all conducted study investigations were performed in accordance with the declaration of Helsinki [23].

\section{Magnetic resonance imaging and analysis}

All participants underwent two MRI scans (BL and after 1 year \pm 3 months) on a 3-T Siemens Tim Trio scanner (Siemens Healthcare GmbH, Erlangen, Germany). 3D T1-weighted images with $1.0 \mathrm{~mm}$ isotropic resolution were collected (MP RAGE, echo time $=2,98 \mathrm{~ms}$, repetition time $=2300 \mathrm{~ms}$, flip angle $=9^{\circ}$, acquisition matrix $=256 \times 256$, number of excitations $=1$, field of view $=256 \mathrm{~mm}$ ). MRI sequences were visually examined (JC; SK) to exclude relevant confounders such as movement artefacts or additional/differential diagnoses such as vascular lesions.

\section{Morphometric measurements}

Morphometric measurements were manually assessed using 3D Slicer Version 4.10.2 (slicer.org). Midsagittal T1-weighted individual MRIs were used for the midbrain and pons measurements, using a simplified version of the methodology described by Massey et al. (2013) [17]. Two independent investigators (JC; SK) blinded to the diagnoses performed the analyses. Each investigator drew line measurements over pons and midbrain (maximal widths perpendicular to the visually estimated oblique superior-inferior axes) in a midsagittal slice to assess the respective area widths. In line with previous research, pons measurements did not include the pontine tegmentum and midbrain measurements did not include the collicular plate [17, 18]. The MTPR was calculated from the determined values dividing the width of the midbrain by the width of the pons for every individual. The rel $\Delta_{\mathrm{t}}$ MTPR was defined as rel $\Delta_{t-} M T P R=\frac{\left(M T P R_{1 Y}-M T P R_{B L}\right)}{M T P R_{B L}}$ with $M T P R_{B L}$ as baseline MTPR and $M T P R_{1 Y}$ as MTPR after 1 year.

\section{Statistical analyses}

All data were analyzed using IBM SPSS version 25.0 (IBM SPSS Statistics, Armonk, NY: IBM Corp.) and R version 3.6.3 (R Foundation for Statistical Computing, Vienna, Austria). Data were evaluated for normality using the Shapiro Wilk test. Parametric, non-parametric or Chi-squared tests were used for group comparisons, depending on the distribution of variables.

To assess inter-rater reliability (IRR) the measurements for midbrain and pons were analyzed using intraclass correlation coefficients (ICCs; single measures, consistency).

Between-group comparisons were performed with an unpaired t-test for DD at BL, Kruskal-Wallis analysis of variance (ANOVA) for age at BL and a Chi-squared test for gender distribution. A multivariate analysis of variance (MANOVA) was performed to investigate differences in midbrain, pons, and MTPR between groups and MRI time points. We calculated a combined parameter of $\mathrm{MTPR}_{\mathrm{BL}}$ and rel $\Delta_{\mathrm{t}-} \mathrm{MTPR}\left(\mathrm{MTPR}_{\mathrm{BL} \& \Delta \mathrm{t}}\right)$ using predicted probability values from a binary logistic regression model. This new parameter was used as test variable in ROC analyses. Thus, it could be estimated whether the diagnostic accuracy of $\mathrm{MTPR}_{\mathrm{BL}}$ can be significantly enhanced by adding rel $\Delta_{\mathrm{t}}$ MTPR. Receiver operating characteristic curve (ROC) analyses were then performed to evaluate the predictive value of the MTPR, rel $\Delta_{\mathrm{t}} \mathrm{MTPR}$, and MTPR $\mathrm{BL}_{\mathrm{B} \Delta \mathrm{t}}$ by computing the area under the curve (AUC; 95\% CI). Diagnostic accuracy was determined in differentiating PSP vs. IPD vs. HC using the optimal cutoff value determined by ROC analysis with $95 \%$ confidence intervals. The cutoff was defined as the value resulting in the highest Youden Index. ROC curves were analyzed for significant differences using the roc.test command of the pROC package [24] in R (method=bootstrap, paired). We also performed a McNemar's test, to evaluate statistical differences in specificity values. A $p$ value $<0.05$ was considered significant for all tests. For all 
statistical comparisons, post-hoc Bonferroni analyses were performed, to correct for multiple comparisons.

\section{Results}

Group characteristics and main findings for PSP, IPD, and $\mathrm{HC}$ are summarized in Table 1 and Figs. 1 and 2. The observed groups did not significantly differ in gender distribution, age, and DD at BL.

\section{Inter-rater reliability}

ICCs revealed good IRR for pons measurements at BL (ICC $=0.87, p<0.001)$, as well as after 1 year $(\mathrm{ICC}=0.89$, $p<0.001)$. Moreover, there was an excellent IRR for midbrain measurements at $\mathrm{BL}(\mathrm{ICC}=0.97, p<0.001)$ and after 1 year $(\mathrm{ICC}=0.98, p<0.001)$. Given this high degree of consistency, averaged values from both raters for midbrain and pons were used to calculate $\mathrm{MTPR}_{\mathrm{BL}}$ and $\mathrm{MTPR}_{1 \mathrm{Y}}$.

\section{Morphometric analyses}

Most collected midbrain MRI measures revealed significant differences between PSP and non-PSP groups: PSP-patients had a smaller $\operatorname{MTPR}_{\mathrm{BL}}(M=0.45 \pm 0.06)$ as well as $\mathrm{MTPR}_{1 \mathrm{Y}}$ $(M=0.43 \pm 0.06)$ compared to both non-PSP groups ( $\mathrm{F}(2$, $42)=66.87, p<0.001$; Fig. 1a). This difference was particularly driven by a smaller midbrain width (MW) at both time points in PSP-patients $(\mathrm{F}(2,42)=60.08, p<0.001)$, whereas the MTPR and MW did not differ between IPD and HC. Furthermore, there was a significant decline in MTPR $(\mathrm{t}(14)=.4 .06, p=0.001)$ over the 1 -year period for the PSP group. The rel $\Delta_{\mathrm{t}-}$ MTPR was stronger in PSP compared to IPD $(p<0.001$; Fig. 1b) with a mean decline of $4.7 \%$ in MTPR for PSP-patients. Conversely, the rel $\Delta_{\mathrm{t}}$ MTPR did not differ between PSP $(M=-4.74 \pm 4.48)$ and $\mathrm{HC}(M=-1.58 \pm 2.18)$. Here, the $\mathrm{MTPR}_{1 \mathrm{Y}}$ in $\mathrm{HC}$ was significantly smaller compared to the corresponding $\mathrm{MTPR}_{\mathrm{BL}}$ ( $\mathrm{t}(29)=2.92, p=0.011)$, which was not the case for patients with IPD. Pontine values did not differ between groups and time points (see Table 1 for statistical details).

\section{ROC analyses}

ROC analyses confirmed excellent diagnostic accuracy for the $\mathrm{MTPR}_{\mathrm{BL}}(\mathrm{AUC}=0.98,95 \%$ CI 0.94-1.00, sensitivity $=93.33 \%$, specificity $=93.33 \%$, accuracy $=93.33 \%$ ) when comparing PSP and IPD-patients (Fig. 2a), as well as PSP and both non-PSP groups ( $\mathrm{AUC}=0.98,95 \% \mathrm{CI}$ $0.94-1.00$, cutoff $\leq 0.540$, sensitivity $=93.33 \%$, specificity $=96.67 \%$, accuracy $=95.56 \%$; Fig. 2b). Regarding rel $\Delta_{\mathrm{t}}$ MTPR accuracy for distinguishing PSP from IPD was good (AUC $=0.85,95 \%$ CI $0.72-0.98$, cutoff $\geq 0.015$, sensitivity $=73.33 \%$, specificity $=80.00 \%$, accuracy $=76.67 \%$; Fig. 2c); Moderate diagnostic accuracy could be demonstrated for distinguishing PSP from non-PSP participants $(\mathrm{AUC}=0.78,95 \%$ CI $0.63-0.93$, sensitivity $=73.33 \%$, specificity $=56.67 \%$, accuracy $=62.22 \%$; Fig. $2 \mathrm{~d}$ ). There was also excellent diagnostic accuracy for $\mathrm{MTPR}_{\mathrm{BL} \& \Delta \mathrm{t}}$ (AUC $=0.97,95 \%$ CI $0.93-1.00$, sensitivity $=93.33 \%$, specificity $=100.00 \%$, accuracy $=96.67 \%$; Fig. 2e) when comparing PSP and IPD as well as for comparing PSP and non-PSP subjects (AUC $=0.98,95 \%$ CI $0.92-1.00, \mathrm{OC} \geq 0.520$, sensitivity $=93.33 \%$, specificity $=100.00 \%$, accuracy $=97.77 \%$; Fig. 2f). There was no significant difference for the diagnostic accuracy of MTPR $\mathrm{BL} \& \Delta \mathrm{t}_{\text {and }}$ aTPR $\mathrm{BL}_{\mathrm{BL}}(D=-0.43$, $p=0.67$ ). Additionally, the specificity values did not differ significantly ( $p=0.500$; see Table 1 for all detailed values).

\section{Discussion}

This is the first study to investigate the MTPR in a longitudinal setting to the best of our knowledge. Our cross-sectional results confirmed a lower MTPR $_{\mathrm{BL}}$ in PSP, when compared both to IPD and to $\mathrm{HC}$; an $\mathrm{MTPR}_{\mathrm{BL}} \leq 0.54$ was indicative of PSP. Longitudinal evidence revealed a distinct 1-year decline of MTPR in PSP-patients, representing a more pronounced midbrain atrophy rate compared to IPD. The combined $\mathrm{MTPR}_{\mathrm{BL} \& \Delta \mathrm{t}}$ slighty improved the already high diagnostic accuracy of $\mathrm{MTPR}_{\mathrm{BL}}$ and likewise improved the specificity to $100 \%$; however, these improvements were not statistically significant.

Overall, MTPR values confirm previous findings suggesting an MTPR $\leq 0.52$ as highly specific for PSP $[17,18]$. Longitudinal results also tally with former research, as differentiation of PSP from IPD is based on studies demonstrating that PSP presents stronger and faster midbrain atrophy [25-27]. However, we were not able to discriminate between PSP and non-PSP groups solely by means of rel $\Delta_{t}$ MTPR as with the $\mathrm{MTPR}_{\mathrm{BL}}$. Still, there was good predictive accuracy for distinguishing PSP and IPD only.

Most importantly, we found increased specificity values for the combined parameter MTPR $\mathrm{BL \&}_{\Delta \mathrm{t}}$. This is of particular clinical relevance considering that a high degree of specificity is very important for distinguishing between various forms of diseases [7]. However, a statistical comparison of specificity values did not reach significance. As the MTPR $\mathrm{BL}_{\mathrm{BL}}$ provided already excellent specificity with $96.67 \%$, when comparing PSP and non-PSP groups, it is hard to improve specificity further in fact. However, with $M_{T P R} R_{B L \& t}$ specificity reached $100 \%$.

With a mean DD of 63.1 months we investigated patients in rather progressed disease stages. This is particularly important considering that midbrain atrophy could also 


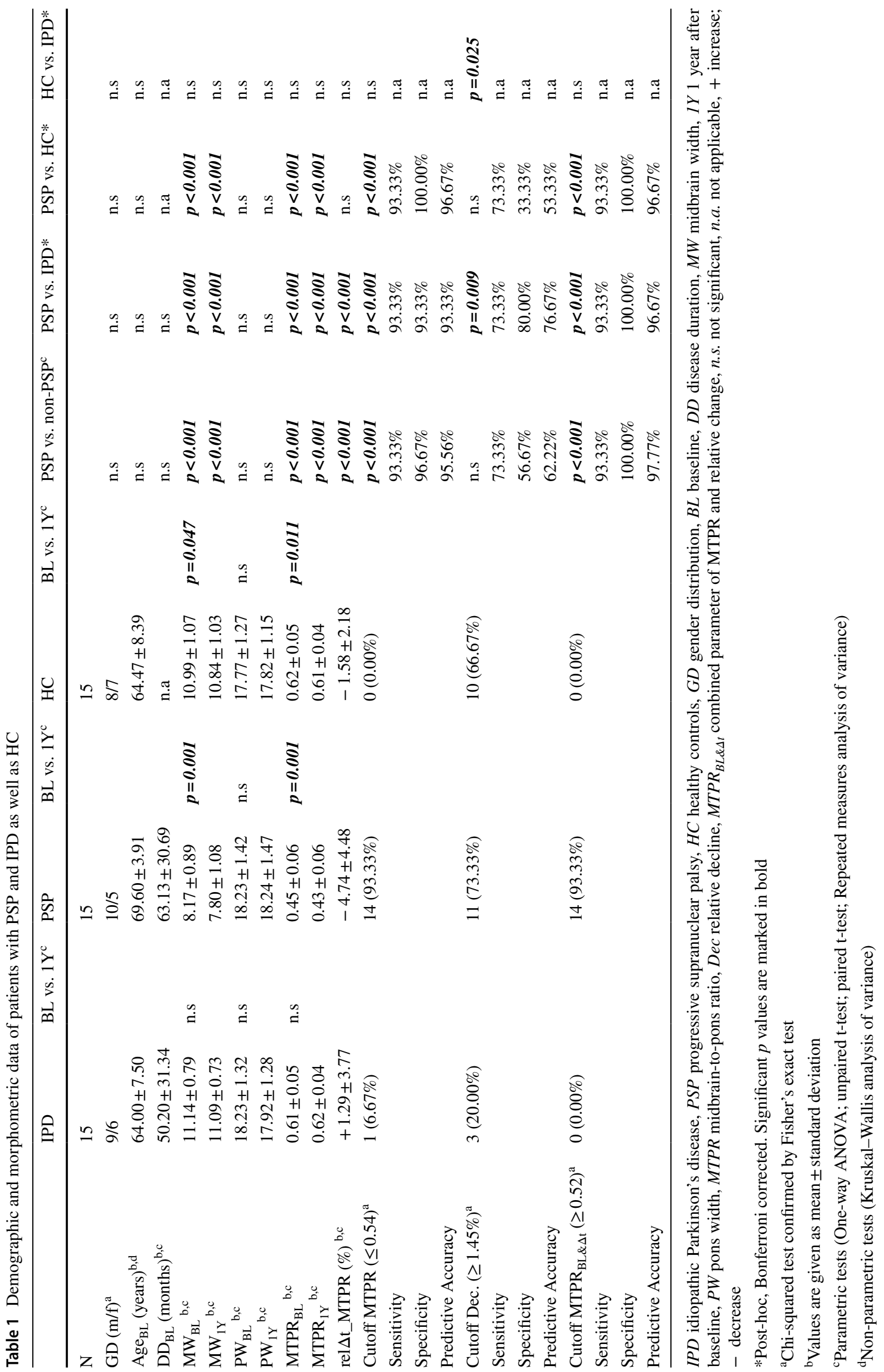




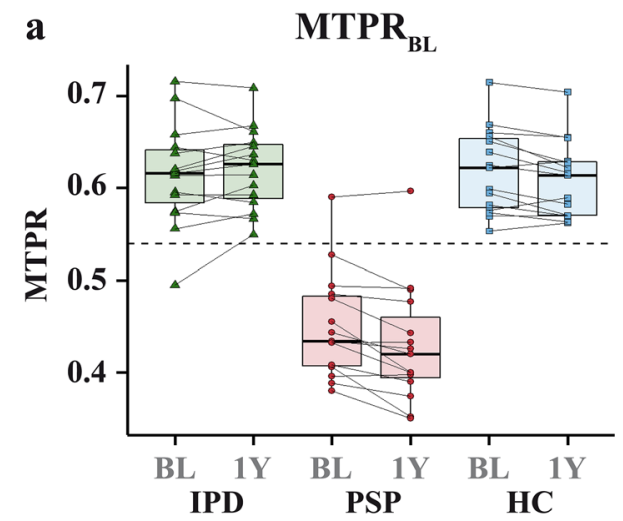

Fig. 1 Main results for the observed groups. a MTPR for all groups at both time points; b rel $\Delta_{\mathrm{t}-}$ MTPR (decline/increase; \%) for all groups. MTPR midbrain-to-pons ratio, IPD idiopathic Parkinson's disease, $P S P$ progressive supranuclear palsy, $H C$ healthy controls,

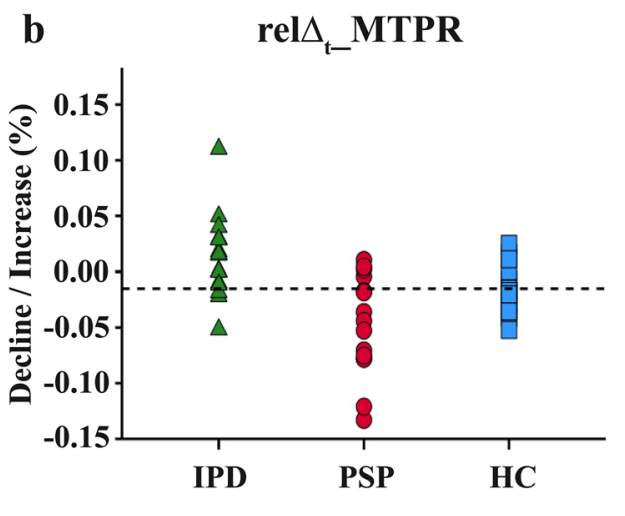

$B L$ Baseline, $1 Y 1$ year after baseline, rel $\Delta t_{-} M T P R$ relative change, $M T P R_{B L \& \Delta t}$ combined parameter of $\mathrm{MTPR}_{\mathrm{BL}}$ and rel$\Delta_{\mathrm{t} \_} \mathrm{MTPR},+$ increase; - decrease a

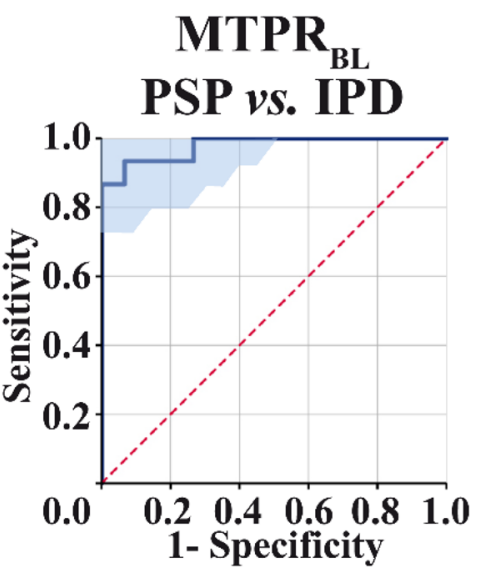

b

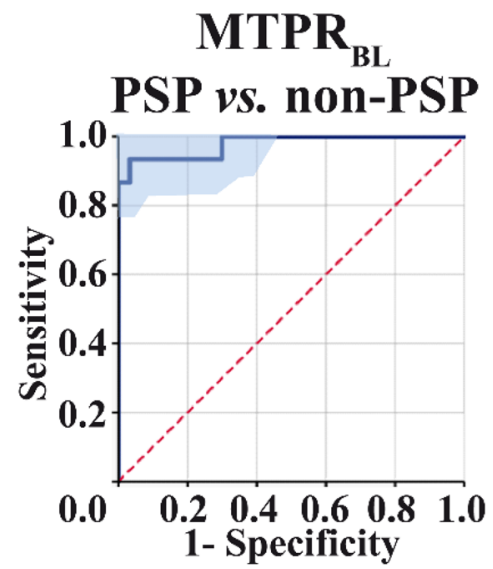

c
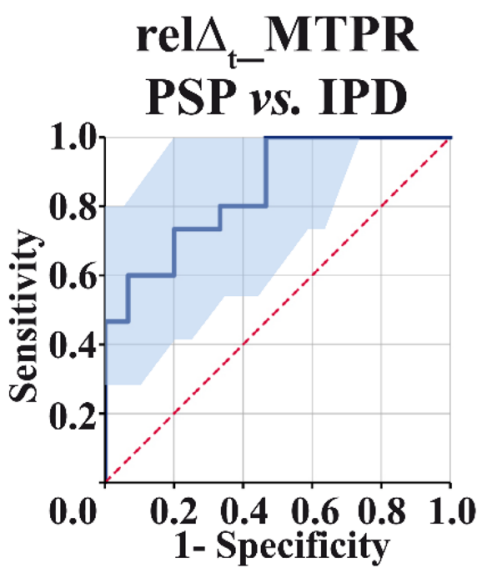

d
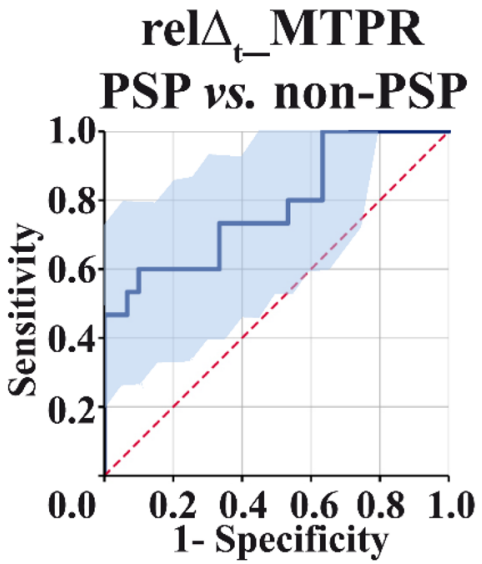

e

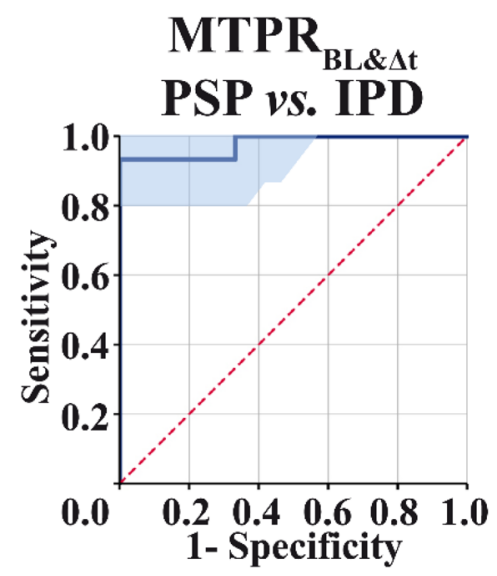

f

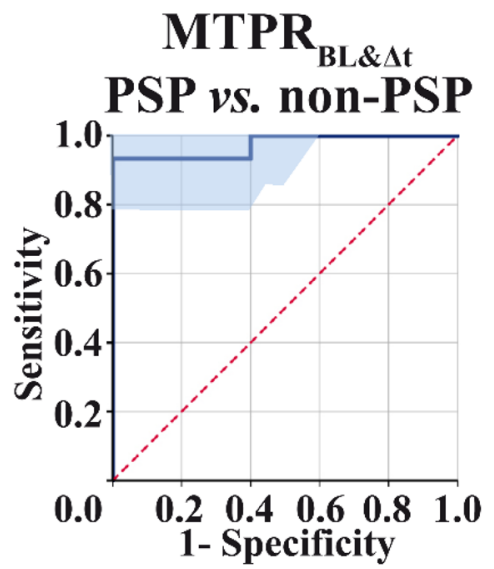

Fig. 2 Main results of ROC analyses. ROC curve for $M_{T P R}$ comparing a PSP vs. IPD and b PSP vs. non-PSP; $\mathbf{c}$ ROC curve for rel $\Delta_{\mathrm{t}}$ MTPR comparing PSP vs. IPD and d PSP vs. non-PSP; $\mathbf{e}$ ROC curve for $M_{T P R} R_{B L \Delta t}$ comparing PSP vs. IPD and f PSP vs.
non-PSP. MTPR midbrain-to-pons ratio, IPD idiopathic Parkinson's disease $P S P$ progressive supranuclear palsy, $H C$ healthy controls, $B L$ Baseline, $I Y 1$ year after baseline, rel $\Delta t_{-} M T P R$ relative change, $M T P R_{B L \& \Delta t}$ combined parameter of $\mathrm{MTPR}_{\mathrm{BL}}$ and rel $\Delta_{\mathrm{t}} \mathrm{MTPR}$ 
serve as PSP-specific preclinical marker in very early disease stages $[28,29]$. Thus, it remains to be studied, whether the rel $\Delta_{\mathrm{t}-} \mathrm{MTPR}$ - as indicator of midbrain atrophy rate-contributes better to diagnostic accuracy, earlier in the disease, i.e., when the overall MTPR has not yet reached PSP-specific values. Again, a larger patient cohort, e.g., from a future multi-centric study, would allow a more detailed analysis of effects of DD, age, and gender. While rel $\Delta_{t}$ MTPR was of limited diagnostic value in our patient cohort with advanced stages of the diseases, it might have a more valuable impact in early stages of PSP, where higher MTPR ratios can be expected.

Moreover, it has to be considered that atrophy rates might differ between different disease stages in PSP, as it was already reported for other neurodegenerative diseases [30]. Additionally, we did not include a quantitative measure of disease severity such as the Progressive Supranuclear Palsy Rating Scale or MDS-Unified Parkinson's Disease Rating Scale. This would have been helpful in estimating disease progression independently from DD.

Patients in this study were diagnosed clinically by expert evaluation; however, misdiagnoses cannot be excluded in the absence of post mortem verification. However, all diagnoses were based on valid diagnostic criteria [21] enhancing the reliability of the clinical diagnosis. Additionally, we have also attempted to retrospectively apply the MDS diagnostic criteria for PSP [22] to allow a more precise description of diagnosis. Four patients, were confirmed to be correctly classified as PSP, by post mortem diagnosis.

In our study, we also did not distinguish between PSPsubtypes, as reported patients were mostly diagnosed with PSP-Richardson`s syndrome. However, it cannot be excluded that distinct subtypes could also differ in atrophy rates, which again points out the importance of further analyses in larger samples.

Moreover, HC presented a decline in MTPR, too. Midbrain shrinkage has been found in healthy ageing previously and, therefore, may have contributed to MTPR reduction, as a significant decline of MW could also be observed [31,32]. Still, midbrain atrophy is assumed to be more pronounced in PSP. Hence, it should be considered, if intersubject variability in PSP could also account for the results at hand.

The important new finding from the present work apart from the confirmation and replication of previous studies on this topic is that specificity values increase by adding the rel $\Delta_{\mathrm{t}}$ MTPR to MTPR $\mathrm{BL}_{\mathrm{BL}}$. This is of high clinical relevance in disease differentiation. Statistical comparison of specificity values did not reach significance, as the $\mathrm{MTPR}_{\mathrm{BL}}$ already had very good values, which were difficult to improve. Still, we believe that the MTPR $\mathrm{BL \& \Delta t}_{\mathrm{t}}$ can further substantiate diagnosis of PSP in follow-up examinations and serve as an additional biomarker of PSP-specific disease progression, which may of particular importance to reveal the efficacy of potential disease-modifying treatments. Finally, the current findings motivate larger patient studies including PSP subtypes and other forms of atypical Parkinsonian diseases to explore the full potential of MTPR and its change as diagnostic tool.

Author contributions SK was involved in execution of the research project, design and execution of statistical analysis and writing of the first draft. JC was involved in execution of the research project, review and critique on statistical analysis and review and critique on the manuscript. LD was involved in execution of the research project, review and critique on statistical analysis and review and critique on the manuscript. ASM was involved involved in execution of the research project, review and critique on statistical analysis and review and critique on the manuscript. SF was involved in execution of the research project, review and critique on statistical analysis and review and critique on the manuscript. MS was involved in conception, organization and execution of the research project. MB was involved in review and critique on statistical analysis and review and critique on the manuscript. AS was involved in conception and organization of the research project, review and critique on statistical analysis and review and critique on the manuscript. CJH was involved in conception, organization and execution of the research project, review and critique on statistical analysis and review and critique on the manuscript.

Funding Open Access funding enabled and organized by Projekt DEAL. The project underlying this report "Helmholtz Alliance for Mental Health in an Ageing Society" (HelMA, HA-215) was supported within the framework of the "Helmholtz Impuls- und Vernetzungsfonds" funded by the German "Bundesministerium für Bildung und Forschung" (BMBF). Furthermore, this work was supported by the "Stiftung für Altersforschung" and the "Forschungskommission", Heinrich-Heine-University, Düsseldorf (Germany).

Data availability The participants of this study did not agree for their data to be shared publicly. Hence, we are not able to offer them for further usage.

\section{Compliance with ethical standards}

Conflicts of interest The authors declare that they have no conflict of interest.

Ethical approval The study was approved by the local ethics committee (study no. 2849).

Consent to participate All participants provided written informed consent prior to enrolment in the study.

Consent for publication All participants provided written informed consent for publication of the study results.

Open Access This article is licensed under a Creative Commons Attribution 4.0 International License, which permits use, sharing, adaptation, distribution and reproduction in any medium or format, as long as you give appropriate credit to the original author(s) and the source, provide a link to the Creative Commons licence, and indicate if changes were made. The images or other third party material in this article are included in the article's Creative Commons licence, unless indicated otherwise in a credit line to the material. If material is not included in the article's Creative Commons licence and your intended use is not permitted by statutory regulation or exceeds the permitted use, you will 
need to obtain permission directly from the copyright holder. To view a copy of this licence, visit http://creativecommons.org/licenses/by/4.0/.

\section{References}

1. Williams DR, de Silva R, Paviour DC et al (2005) Characteristics of two distinct clinical phenotypes in pathologically proven progressive supranuclear palsy: Richardson's syndrome and PSPparkinsonism. Brain 128(6):1247-1258

2. Litvan I (2003) Update on epidemiological aspects of progressive supranuclear palsy. Mov Disord 18(Suppl. 6):43-50

3. McFarland NR (2016) Diagnostic approach to atypical parkinsonian syndromes. CONTINUUM Lifelong Learn Neurol 22(4 Movement disorders): 1117

4. Hughes AJ, Daniel SE, Kilford L, Lees AJ (1992) Accuracy of clinical diagnosis of idiopathic Parkinson's disease: a clinicopathological study of 100 cases. J Neurol Neurosurg Psychiatry $55: 181-184$

5. Jankovic J (2008) Parkinson's disease: clinical features and diagnosis. J Neurol Neurosurg Psychiatry 79(4):368-376

6. Poewe W, Wenning G (2002) The differential diagnosis of Parkinson's disease. Eur J Neurol 9:23-30

7. van Eimeren T, Antonini A, Berg D et al (2019a) Neuroimaging biomarkers for clinical trials in atypical parkinsonian disorders: proposal for a neuroimaging biomarker utility system. Alzheimer's Dement Diagn Assess Dis Monit 11:301-309

8. Seppi K, Poewe W (2010) Brain magnetic resonance imaging techniques in the diagnosis of parkinsonian syndromes. Neuroimaging Clin 20(1):29-55

9. Quattrone A, Nicoletti G, Messina D et al (2008) MR imaging index for differentiation of progressive supranuclear palsy from Parkinson disease and the Parkinson variant of multiple system atrophy. Radiology 246:214-221

10. Mahlknecht P, Hotter A, Hussl A, Esterhammer R, Schocke M, Seppi K (2010) Significance of MRI in diagnosis and differential diagnosis of Parkinson's disease. Neurodegener Dis 7(5):300-318

11. Stamelou M, Knake S, Oertel WH et al (2011) Magnetic resonance imaging in progressive supranuclear palsy. J Neurol 258(4):549-558

12. Schrag A, Good CD, Miszkiel K et al (2000) Differentiation of atypical parkinsonian syndromes with routine MRI. Neurology 54:697-702

13. Focke NK, Helms G, Pantel PM et al (2011) Differentiation of typical and atypical Parkinson syndromes by quantitative MR imaging. Am J Neuroradiol 32(11):2087-2092

14. Kato N, Arai K, Hattori T (2003) Study of the rostral midbrain atrophy in progressive supranuclear palsy. J Neurol Sci 210(1-2):57-60

15. Mueller C, Hussl A, Krismer F et al (2018) The diagnostic accuracy of the hummingbird and morning glory sign in patients with neurodegenerative parkinsonism. Parkinsonism Relat Disord 54:90-94

16. Oba H, Yagishita A, Terada $\mathrm{H}$ et al (2005) New and reliable MRI diagnosis for progressive supranuclear palsy. Neurology 64:2050-2055
17. Massey LA, Micallef C, Paviour DC et al (2012) Conventional magnetic resonance imaging in confirmed progressive supranuclear palsy and multiple system atrophy. Mov Disord 27(14):1754-1762

18. Kaasinen V, Kangassalo N, Gardberg M et al (2015) Midbrain-topons ratio in autopsy-confirmed progressive supranuclear palsy: replication in an independent cohort. Neurol Sci 36(7):1251-1253

19. Josephs KA, Whitwell JL, Boeve BF et al (2006) Rates of cerebral atrophy in autopsy-confirmed progressive supranuclear palsy. Ann Neurol 59(1):200-203

20. Hwang M, Yang H, Kim Y et al (2017) (2013) Differential progression of midbrain atrophy in parkinsonism: longitudinal MRI study. Neurodegener Dis 17(1):31-37

21. Litvan I, Agid Y, Calne D et al (1996) Clinical research criteria for the diagnosis of progressive supranuclear palsy (SteeleRichardson-Olszewski syndrome): report of the NINDS-SPSP international workshop. Neurology 47:1-9

22. Höglinger GU, Respondek G, Stamelou M et al (2017) Clinical diagnosis of progressive supranuclear palsy: the movement disorder society criteria. Mov Disord 32:853-864

23. World Medical Association (2013) World Medical Association Declaration of Helsinki: ethical principles for medical research involving human subjects. JAMA 310:2191-2194

24. Robin X, Turck N, Hainard A et al (2011) pROC: an open-source package for $\mathrm{R}$ and $\mathrm{S}+$ to analyze and compare ROC curves. BMC Bioinform 12:77

25. Paviour DC, Price SL, Jahanshahi M, Lees AJ, Fox NC (2006) Regional brain volumes distinguish PSP, MSA-P, and PD: MRIbased clinico-radiological correlations. Mov Disord Off J Mov Disord Soc 21(7):989-996

26. Warmuth-Metz M, Naumann M, Csoti I, Solymosi L (2001) Measurement of the midbrain diameter on routine magnetic resonance imaging: a simple and accurate method of differentiating between Parkinson disease and progressive supranuclear palsy. Arch Neurol 58:1076-1079

27. Dutt S, Binney RJ, Heuer HW et al (2016) Progression of brain atrophy in PSP and CBS over 6 months and 1 year. Neurology 87(19):2016

28. Ahn JH, Kim M, Kim JS et al (2019) Midbrain atrophy in patients with presymptomatic progressive supranuclear palsy-Richardson's syndrome. Parkinsonism Relat Disord 66:80-86

29. Owens E, Krecke K, Ahlskog JE et al (2016) Highly specific radiographic marker predates clinical diagnosis in progressive supranuclear palsy. Parkinsonism Relat Disord 28:107-111

30. Fiford CM, Ridgway GR, Cash DM et al (2018) Patterns of progressive atrophy vary with age in Alzheimer's disease patients. Neurobiol Aging 63:22-32. https://doi.org/10.1016/j.neurobiola ging.2017.11.002

31. Lambert C, Chowdhury R, Fitzgerald T et al (2013) Characterizing aging in the human brainstem using quantitative multimodal MRI analysis. Front Hum Neurosci 7:462

32. Luft AR, Skalej M, Schulz JB et al (1999) Patterns of age-related shrinkage in cerebellum and brainstem observed in vivo using three-dimensional MRI volumetry. Cereb Cortex 9(7):712-721 